Ann. Funct. Anal. 6 (2015), no. 4, 107-113

http://doi.org/10.15352/afa/06-4-107

ISSN: 2008-8752 (electronic)

http://projecteuclid.org/afa

\title{
EKELAND'S VARIATIONAL PRINCIPLE AND CRITICAL POINTS OF DYNAMICAL SYSTEMS IN LOCALLY COMPLETE SPACES
}

\author{
C. BOSCH$^{1 *}$, C.L. GARCÍA ${ }^{1}$, F. GARIBAY-BONALES ${ }^{2}$, C. GÓMEZ-WULSCHNER ${ }^{1}$ \\ AND R. VERA ${ }^{2}$ \\ Communicated by K. Ciesielski
}

\begin{abstract}
Ekeland's variational principle and the existence of critical points of dynamical systems, also known as multiobjective optimization, have been proved in the setting of locally complete spaces. In this article we prove that these two properties can be deduced one from the other under certain convexity conditions.
\end{abstract}

\section{INTRODUCTION AND PRELIMINARIES}

One of the most important tools to solve problems in optimization, optimal control, game theory, nonlinear equations, dynamical systems, etc., is the Ekeland's variational principle [5]. Since the discovery of such a principle there have also appeared many extensions or equivalent formulations of the principle [2], or [10]. In [2] C.Bosch, A. García, C.L.García, established the variational principle in the setting of locally complete spaces.

In 1983 G.Isaac [8] obtained a general existence result considering a set as a critical point for a dynamical system and using the completeness of the space. By adapting some ideas of the cited paper, C. Bosch , A. García in [3] extended that multiobjective optimization result to locally convex spaces by assuming that some related sets are locally complete.

Date: Received: Feb. 14 2015; Accepted: Mar. 23, 2015.

* Corresponding author.

2010 Mathematics Subject Classification. Primary 46N10; Secondary 47N10, 49J53.

Key words and phrases. Ekeland's variational principle, locally complete space, multiobjective optimization, absolutely convex. 
In the setting of locally complete spaces we prove that Ekeland's variational principle and multiobjective optimization can be derived from each other, making them, in this sense equivalent.

Throughout this paper $(E, \tau)$ will denote a locally convex space with topology $\tau$, generated by a family of seminorms $\left\{\rho_{\alpha}: \alpha \in \Lambda\right\}$ with $\Lambda$ a set of indices. A disk $B$ in $\mathrm{E}$ is a closed, bounded and absolutely convex set. We denote by $\left(E_{B}, \rho_{B}\right)$ the linear span of $B$ endowed with the topology defined by the Minkowski functional associated with $B$. When $B$ is bounded, $\rho_{B}$ is a norm, and the norm topology is finer than the topology inherited from E. If $\left(E_{B}, \rho_{B}\right)$ is a Banach space we say that $B$ is a Banach disk. $E$ is a locally complete locally convex space (briefly locally complete space) if each closed, bounded disk is a Banach disk. There are many examples of locally complete spaces, in fact every sequentially complete space is locally complete. On the other side one can also find examples of locally complete spaces that are not sequentially complete. A sequence $\left(x_{n}\right)$ in $E$ is said to be locally convergent or Mackey convergent to an element $x$ of $E$, if there exists a disc $B$ in $E$ such that the sequence converges to $x$ in $E_{B}$ with respect to $\rho_{B}$. A sequence is called locally Cauchy or Mackey Cauchy if it is a $\rho_{B}$-Cauchy sequence in $E_{B}$ for a certain disk in $E$. Let $C$ be a non void subset of $E$. A point $x$ is a local limit point of $C$ if there is a sequence in $C$ that is locally convergent to $x$. A set $C$ is locally closed if every local limit point of $C$ belongs to $C$. A subset $A$ of a space $E$ is said to be locally complete if every locally Cauchy sequence in $A$ converges locally to a point of $A$. It is clear that every locally complete subset of a space is locally closed.

In [9] [2-14 p.20] locally complete spaces are also given the name $c^{\infty}$ - or convenient spaces. It should be noted that the structure of $c^{\infty}$ - (locally complete) spaces have become important in recent years due to the use of such spaces in nonlinear distribution theory. More detail about applications of these spaces can be found in [9] and the references therein. In [6] one can also see how theorems on existence and uniqueness for integro-differential equations are now extended to locally complete spaces.

The class $\Phi$ of perturbations we will use, is defined as the family of functions $\varphi:[0, \infty) \rightarrow[0, \infty)$ which are subadditive, strictly increasing, continuous and such that $\varphi(0)=0$, and $\lim _{x \rightarrow \infty} \varphi(x)=\infty$. Clearly the inverse of $\varphi$ exists and is superadditive, strictly increasing and continuous, $\varphi^{-1}(0)=0$. Here $\varphi$ is said to be subadditive if $\varphi(s+t) \leq \varphi(s)+\varphi(t)$, for every $s, t \in[0, \infty)$, and $\varphi^{-1}$ is said to be superadditive if $\varphi^{-1}(s+t) \geq \varphi^{-1}(s)+\varphi^{-1}(t)$, for every $s, t \in[0, \infty)$. Functions like $\varphi(t)=t, \varphi(t)=\sqrt[n]{t}, \varphi(t)=\ln (1+t)$, are examples of elements in $\Phi$.

A closed, pointed convex cone in a locally convex space is a nonempty subset $K \subset$ E such that:

(1) $K$ is a closed subset,

(2) $K+K \subseteq K$

(3) $\lambda K \subseteq K$, for all $\lambda \in \mathbf{R}$

(4) $K \cap(-K)=\{0\}$ 
If a closed, pointed convex cone $K \subset E$ is given we can define an ordering in $E$ by $x \preceq y$ if and only if $y-x \in K$.

If $A \subset E$, is a nonempty subset we say that $a \in A$ is an efficient (maximal) point of $A$, with respect to $K$, if $A \cap(a+K)=\{a\}$. We denote by $E(A ; K)$ the set of efficient points of $A$ with respect to $K$.

We say that $\Gamma: A \rightarrow 2^{A}$ is a dynamical system if for every $x \in A$, and $\Gamma(x)$ is a nonempty subset of $A$ and $x^{*} \in A$ is a critical point for $\Gamma$ if $\Gamma\left(x^{*}\right)=\left\{x^{*}\right\}$. We can see easily that $\Gamma_{A}(x)=A \cap(x+K)$ for every $x \in A$ is a dynamical system. The reader can verify that an element $x^{*} \in A$ is an efficient point of $A$, if and only if $x^{*}$ is a critical point of $\Gamma_{A}$.

A function $\Psi: A \rightarrow \mathbf{R} \cup\{+\infty\}$ is proper if it is not identically $+\infty$. A proper function $\Psi: A \rightarrow \mathbf{R} \cup\{+\infty\}$ is (locally) lower semicontinuous if $\{x \in A$ : $\Psi(x) \leq r\}$ is (locally) closed in $E$ for every $r$ in $\mathbf{R}$ or equivalently for each $x^{\prime} \in A$ and every sequence $\left(x_{n}\right)$ in $A$, (locally) convergent to $x^{\prime}$ we have that $\Psi\left(x^{\prime}\right) \leq \liminf \Psi\left(x_{n}\right)$.

\section{MAin RESUlts}

In this section we present an equivalence between Ekeland's variational principle for locally complete spaces [2] and critical points of dynamical systems or multiobjective optimization [3]. To do that we need a technical condition on certain sets. First we will state two theorems, the third one will give the conditions under which they are equivalent.

Theorem 2.1. Let $(E, \tau)$ be a locally complete space and $f: E \rightarrow \mathbf{R} \cup\{\infty\}$ be a proper, lower semicontinuous and bounded below function. Let $\varphi$ be in $\Phi$ and $x_{0}$ be a point in Dom $(f)$, that is $f\left(x_{0}\right)<\infty$. Then for any Banach disk $B$ in $E$ such that $x_{0} \in E_{B}$ there exists $x^{*} \in E_{B}$ such that:

(a) $f\left(x^{*}\right)+\varphi\left(\rho_{B}\left(x^{*}-x_{0}\right)\right) \leq f\left(x_{0}\right)$ and

(b) $f\left(x^{*}\right)<\varphi\left(\rho_{B}\left(x^{*}-x\right)\right)+f(x)$ for all $x \in E_{B} \backslash\left\{x^{*}\right\}$

S. Al Homidan, Q.H. Ansari and J.C. Yao established in [7] [corollary 3.1] a similar result to the previous theorem but as they pointed out in their remark 3.3 the results are not comparable, they are of different kind.

Theorem 2.2. Let $(E, \tau)$ be a locally convex space and $\varphi$ be in $\Phi$. Let $A$ be a nonempty locally complete subset of $E$. Let $\Gamma: A \rightarrow 2^{A}$ be a dynamical system. Suppose that there exists $\Psi: A \rightarrow \mathbf{R}$ locally lower semicontinuous and bounded below. If there exists $\left\{c_{\alpha} \in \mathbf{R}^{+}: \alpha \in \Lambda\right\}$ such that:

(a) $D=\cap_{\alpha}\left\{x \in E: c_{\alpha} \rho_{\alpha}(x) \leq 1\right\}$ is a non zero Banach disc.

(b) For every $x \in A$ and for every $u \in \Gamma(x)$ we have $\varphi\left(c_{\alpha} \rho_{\alpha}(x-u)\right) \leq \Psi(x)-$ $\Psi(u)$ for every $\alpha \in \Lambda$.

Then $\Gamma$ has a critical point $x^{*} \in A$.

Theorem 2.3. Theorem 2.1 and 2.2 are equivalent if $A$ is absolutely convex.

Proof. First let us assume that Theorem 2.1 holds and that the hypothesis of Theorem 2.2 are satisfied with $A$ being absolutely convex. Let $E_{A}=\bigcup_{r>0} r A$. Now we have two possibilities ${\overline{D \cap E_{A}}}^{\tau} \neq\{0\}$ or $D \cap E_{A}=\{0\}$. 
Case $1: C={\overline{D \cap E_{A}}}^{\tau} \neq\{0\}$.

By the definition $C$, is $\tau$-closed, bounded since $D$ is bounded, balanced and convex since it is the intersection of two balanced and convex sets $D$ and $E_{A}$. Now since $C \neq\{0\}$ there exists $y_{o} \neq 0$ such that $y_{o} \in D \cap E_{A}$, then there is an $r_{o}$ such that $y_{o} \in D \cap r_{o} A$. If $r_{o} \leq 1$ then $y_{o} \in D \cap A$ and we denote $x_{o}=y_{o} \in D \cap A \subset C$. If $1<r_{o}$ we will take $x_{o}=\frac{1}{r_{o}} y_{o} \in D \cap A \subset C$.

Since $D$ is closed and $C \subset D$ we have that $E_{C} \subset E_{D}$ and $\rho_{D}(x) \leq \rho_{C}(x)$ for every $x \in E_{C}$.

Claim 1: If $\left(E_{D}, \rho_{D}\right)$ is complete then $\left(E_{C}, \rho_{C}\right)$ is also complete.

Let $\left\{x_{n}\right\} \subset E_{C}$ be $\rho_{C}$-Cauchy, then it is also $\rho_{D}$-Cauchy and since we are assuming that $\left(E_{D}, \rho_{D}\right)$ is complete, there exists $x \in E_{D}$ such that $x_{n} \rightarrow x$ with respect to $\rho_{D}$. We have that $\left\{x_{n}\right\} \subset E_{C}$ is $\rho_{C}$-Cauchy so for $\varepsilon>0$ there exists $N \in \mathbf{N}$ such that if $n, m \geq N$ then $x_{n}-x_{m} \in \varepsilon C$. We use that the topology induced by $\rho_{D}$ in $E_{D}$ is stronger than the topology induced in $E_{D}$ by the topology $\tau$ and the fact that $C \subset E_{D}$ is $\tau$-closed to conclude that $\bar{C}^{\rho_{D}}=C$. Then $\rho_{C}\left(x_{n}-x\right)<\varepsilon$ for every $n \geq N$, that is, $x_{n} \rightarrow x \in\left(E_{C}, \rho_{C}\right)$, which proves the claim.

Claim 2: $\rho_{D}(x)=\rho_{C}(x)$ for every $x \in E_{C} \cap E_{A}$.

Take $x$ such that $\rho_{D}(x)<t$ then $x \in t D \cap E_{A}=t\left(D \cap E_{A}\right)=t C$ so $\rho_{C}(x)<t$ which means that $\rho_{C}(x) \leq \rho_{D}(x)$. Now using the fact that $\rho_{D}(x) \leq \rho_{C}(x)$ for every $x \in E_{C}$, we have the equality of the seminorms $\rho_{D}(x)=\rho_{C}(x)$.

Now apply Theorem 2.1 to the complete space $\left(E_{C}, \rho_{C}\right)$, with $B=C, x_{o} \in$ $D \cap A \subset C$ and the function $g: E_{C} \rightarrow R \cup\{\infty\}$ defined by

$$
g(x)= \begin{cases}\Psi(x), & x \in A \\ \infty, & x \in E_{C} \backslash A\end{cases}
$$

$g$ is a lower semicontinuous and bounded below function, since $\Psi$ is locally lower semicontinuous in $A$ and then locally lower semicontinuous $\left(E_{C}, \rho_{C}\right)$, furthermore $\Psi$ is bounded below in $A$. Then there exists $z_{o} \in E_{C}$ such that

(a) $g\left(z_{o}\right)+\rho_{C}\left(x_{o}-z_{o}\right) \leq g\left(x_{o}\right)=\Psi\left(x_{o}\right)<\infty$ and

(b) $g\left(z_{o}\right)<g(x)+\rho_{C}\left(x-z_{o}\right)$ for all $x \in E_{C}, x \neq z_{o}$.

Using (a) we have $g\left(z_{0}\right)<\infty$ which says that $z_{0} \in A$.

Claim 3: $\Gamma\left(z_{o}\right) \bigcap E_{C}=\left\{z_{o}\right\}$.

Take $x \in \Gamma\left(z_{o}\right) \bigcap E_{C}$ and suppose that $x \neq z_{0}$ then $\Psi(x)+\varphi\left(\rho_{D}\left(x-z_{o}\right)\right) \leq$ $\Psi\left(z_{o}\right)<\infty$ using the function $g$ we have $g(x)+\varphi\left(\rho_{C}\left(x-z_{o}\right)\right) \leq g\left(z_{o}\right)$ since for $x \in \Gamma\left(z_{o}\right) \subset g(x)=\Psi(x)$ and for $x-z_{o} \in E_{C} \rho_{D}\left(x-z_{o}\right)=\rho_{C}\left(x-z_{o}\right)$. Finally since $B=C$ using item (b) from Theorem 2.1 we get $g\left(z_{0}\right)<\varphi\left(\rho_{B}\left(z_{0}-x\right)\right)+g(x)$ which gives a contradiction and then $x=z_{0}$.

Claim 4: $\Gamma\left(z_{o}\right) \subset E_{C}$.

Take $x \in \Gamma\left(z_{o}\right) \subset A$ then $x-z_{o} \in E_{D}$ and $x \in z_{o}+E_{D} \subset E_{C}+E_{D}=E_{D}$, so there exists $r \geq 1$ such that $x \in r D$ which means that $\frac{1}{r} x \in D \cap A \subset E_{C}$, and then $x \in r E_{C}=E_{C}$.

To finish the proof note that claim 3 and claim 4 give that $\Gamma\left(z_{o}\right)=\left\{z_{o}\right\}$ so in the case that $C={\overline{D \cap E_{A}}}^{\tau} \neq\{0\}$, Theorem 2.2 holds by taking $x^{*}=z_{o}$.

Case 2: $D \cap E_{A}=\{0\}$. 
Again, $A$ is balanced and convex. Take $x_{o} \in A$ and let $C=\left\{x \in A \mid \rho_{\alpha}(x) \leq\right.$ $\left.\rho_{\alpha}\left(x_{o}\right) \forall \alpha \in \Lambda\right\}, C$ is bounded, balanced, convex and $\tau$-closed, furthermore $x_{o} \in C \subset A$.

Claim 5: $\left(E_{C}, \rho_{C}\right)$ is complete.

Take $\left\{x_{n}\right\} \subset E_{C}$ such that $\left\{x_{n}\right\}$ is $\rho_{C}$-Cauchy then it is $\rho_{C}$-bounded so there exists $r>0$ such that $\left\{x_{n}\right\} \subset r C \subset r A$ then $\left\{\frac{1}{r} x_{n}\right\} \subset A$ is locally Cauchy ( $\rho_{C}$-Cauchy) and then there exists $y \in A \cap E_{C}$ such that $\left\{\frac{1}{r} x_{n}\right\} \rightarrow y$ with respect to $\rho_{C}$ so we have $\left\{x_{n}\right\} \rightarrow x=r y$, with respect to $\rho_{C}$.

Now apply Theorem 2.1 to the complete space $\left(E_{C}, \rho_{C}\right)$, with $B=C, x_{o} \in$ $B$ and the function $g: E_{C} \rightarrow R \cup\{\infty\}$ defined by

$$
g(x)= \begin{cases}\Psi(x), & x \in A \\ \infty, & x \in E_{C} \backslash A\end{cases}
$$

$g$ is a lower semicontinuous and bounded below function, since $\Psi$ has the necessary properties. Then there exists $z_{o} \in E_{C}$ such that

(a) $g\left(z_{o}\right)+\rho_{C}\left(x_{o}-z_{o}\right) \leq g\left(x_{o}\right)=\Psi\left(x_{0}\right)<\infty$

(b) $g\left(z_{o}\right)<g(x)+\rho_{C}\left(x-z_{o}\right)$ for every $x \in E_{C} \backslash\left\{z_{o}\right\}$.

The property (a) says that $z_{o} \in A$.

Claim 6: $\Gamma\left(z_{o}\right) \cap E_{C}=\left\{z_{o}\right\}$.

Take $x \in \Gamma\left(z_{o}\right) \cap E_{C}$ then $x-z_{o} \in E_{D} \cap E_{C}$ and $x-z_{o} \in r_{1} D \cap r_{2} C$, choose $r=\max \left(r_{1}, r_{2}\right)$ so we have,$x-z_{o} \in r D \cap r C \subset r D \cap r A=r(D \cap A)$. This can be rewritten as $\frac{1}{r}\left(x-z_{o}\right) \in D \cap A \subset D \cap E_{A}=\{0\}$ and we can conclude that $x=z_{0}$.

Claim 7: $\Gamma\left(z_{0}\right) \subset E_{C}$

Take $x \in \Gamma\left(z_{o}\right) \subset A$ then $x-z_{o} \in E_{D}$ so $x \in z_{o}+E_{D} \subset E_{C}+E_{D}=E_{D}$, now take $r \geq 1$ such that $x \in r D$ and we have $\frac{1}{r} x \in D \cap A \subset E_{C}$, and conclude that $x \in r E_{C}=E_{C}$.

To finish the proof note that claim 6 and claim 7 give that $\Gamma\left(z_{o}\right)=\left\{z_{o}\right\}$ so in the case that $D \cap E_{A}=\{0\}$, Theorem 2.2 hold by taking $x^{*}=z_{o}$.

Now we prove that Theorem 2.2 implies Theorem 2.1.

Since Theorem 2.2 holds, suppose $(E, \tau)$ is a locally convex space, $\left\{\rho_{\lambda}: \lambda \in \Lambda\right\}$ the family of semi-norms that define the topology $\tau$ and $\varphi$ is in $\Phi$. Let $f: E \rightarrow$ $\mathbf{R} \cup\{\infty\}$ be a proper, lower semicontinuous and bounded below function, such that $f\left(x_{0}\right)<\infty$ for some $x_{0} \in E_{B}$. Take $B$ a disc in $E$, since $(E, \tau)$ is a locally complete then $E_{B}$ is complete, $B$ is a Banach disc, it is bounded and then for each $\lambda \in \Lambda$ there exists $r_{\lambda} \in \mathbf{R}^{+}$such that $\rho_{\lambda}(x) \leq r_{\lambda}$ for every $x \in B$ or $c_{\lambda} \rho_{\lambda}(x) \leq 1$ where $c_{\lambda}=1 / r_{\lambda}$. Let $D=\left\{x \in E: \rho_{\lambda}(x) \leq r_{\lambda}\right.$ for every $\lambda \in \Lambda\}=\cap_{\lambda}\left\{x \in E: c_{\lambda} \rho_{\lambda}(x) \leq 1\right\}$, so by part (a) of theorem $2.2, D$ is a disc and $E_{D}$ is complete. Furthermore $B \subset D$, then $E_{B} \subset E_{D}$ and $\rho_{D}(x) \leq \rho_{B}(x)$ for $x \in E_{B}$.Define $\Gamma: S_{B}\left(x_{0}\right) \rightarrow 2^{S_{B}\left(x_{0}\right)}$ by $\Gamma(x)=S_{B}(x) \subset S_{B}\left(x_{0}\right)=A$ where $S_{B}(x)=\left\{y \in E_{B}: f(y)+\varphi\left(\rho_{B}(y-x)\right) \leq f(x)\right\}$. If $S_{B}\left(x_{0}\right)=\left\{x_{0}\right\}$ then take $x^{*}=x_{0}$ and Theorem 2.1 holds. If not take $x \in E_{B}$ and $u \in \Gamma(x)$, then $c_{\lambda} \rho_{\lambda}(x) \leq \sup \left\{c_{\lambda} \rho_{\lambda}(x): \lambda \in \Lambda\right\}=\rho_{D}(x) \leq \rho_{B}(x)$ we then have $\varphi\left(c_{\lambda} \rho_{\lambda}(x-u)\right) \leq$ $\varphi\left(\rho_{D}(x-u)\right) \leq \varphi \rho_{B}(x-u) \leq f(x)-f(u)$. By defining $\left.f\right|_{A}=\Psi$ we have that $\Psi$ is locally lower semicontinuous since $f$ is lower semicontinuous in $A$ then it is locally lower semicontinuous in $A$, now $\varphi\left(c_{\lambda} \rho_{\lambda}(x-u)\right) \leq \Psi(x)-\Psi(u)$ so by Theorem 
2.2 we can conclude that $\Gamma$ has a critical point $x^{*} \in A$ then $\Gamma\left(x^{*}\right)=\left\{x^{*}\right\}$, and Theorem 2.1 holds by using $x^{*}$.

Note that Theorem 2.2 implies Theorem 2.1 whether $A$ is absolutely convex or not.

\section{Final COMments}

Over more than three decades a good deal of effort has been made to look for equivalent formulations or generalizations of Ekeland's Variational Principle (EVP). In [10] and the references therein one can see the flavor of these generalizations. The main result in the seminal article of Ekeland [5] says, roughly, that for a lower semicontinuous bounded below function $f$ on a complete metric space $X$, a slight perturbation of the function has a strict minimum. In the category of normed spaces, the perturbation is taken as a multiple of the norm. EVP was proved to hold for spaces that are not necessary complete metric spaces but that enjoy completeness and generalize distances or weaker form of completeness in locally convex spaces, these two situations as pointed out before can not be compared. The EVP is proved with the weaker completeness, locally complete spaces in [2]. Furthermore in [4] it is shown that in locally complete spaces EVP is equivalent to Caristi-Kirk type fixed point theorem, Takahashi type minimization theorem and an equilibrium version of EVP. Multiobjective optimization theorem deals with the existence of efficient points. Theorem 2.2 asserts, in a quite general setting, the existence of efficient points. In locally complete spaces we have proved that multiobjective optimization and EVP can be deduced from each other and from the equilibrium version of EVP. In the proof of Theorem 2.3 one can see the relation between the efficient points and the point where the perturbed function attains its minimun. Relations between EVP and the multiobjective optimization have been studied in different contexts, a good reference is [1].

Aknowledgement. The first three authors want to acknowledge the partial support given by the Asociación Mexicana de Cultura A.C.

\section{REFERENCES}

1. H. Attouch and H. Riahi, Results for Ekeland's $\epsilon$-Variational Principle and Cone Extremal Solutions, Math. Oper. Res. 18 (1993), no. 1, 173-201.

2. C. Bosch, A. García and C.L. García, An extension of Ekeland's variational principle to locally complete spaces, J. Math. Anal. Appl. 328 (2007), 106-108.

3. C. Bosch and A. García, Local Completeness and Pareto Optimization, Nonlinear Anal. 73 (2010), 1098-1100 .

4. C. Bosch, A. García, C. Gǿmez-Wulschner and S. Hernández-Linares, Equivalent to Ekeland's Variational Principle in locally complete spaces, Sci. Math. Jpn. 72 (2010), no. 3, 283-287.

5. I. Ekeland, On the variational principle, J. Math. Anal. Appl. 47 (1974), 324-353.

6. T. Gilsdorf and M. Khavanin, Existence and uniqueness for nonlinear integro-differential equations in rel locally complete spaces, Sci. Math. Jpn. 76 no.3 (2013), 395-400.

7. S. Al-Homidan, Q.H. Ansari and J.-C. Yao, Some generalizations of Ekeland-type variational principle with applications to equilibrium problems and fixed point theory, Nonlinear Anal. 69 (2008), 126-139. 
8. G. Isaac, Sur l'existence de l'optimum de Pareto, Riv. Math. Univ. Parma 9 (1983), no. 4, 303-325.

9. A. Kriegl and P.W. Michor, The convenient setting: Global Analysis, AMS, Surveys and monographs 53, 1973.

10. J.H. Qiu, P-distances, $q$-distances and a generalized Ekeland's variational principle in uniform spaces, Acta Math. Sin. (Engl. Ser.) 28 (2012), no. 2, 235-254 .

1 Instituto Tecnológico Autónomo de México, México D.F. 01080, México.

E-mail address: bosch@itam.mx; clgarcia@itam.mx; claudiag@itam.mx

2 Universidad Michoacana de San Nicolás de Hidalgo, Morelia 58030, México

E-mail address: rigovera@gmail.com; fgaribay@gmail.com 